DOI: $10.3901 / J M E .2020 .23 .193$

\title{
基于电场驱动熔融喷射聚合物基复合材料 高分辨率3D打印*
}

\author{
杨 昆张广明 李晓强杨建军 彭子龙 兰红波 \\ (青岛理工大学山东省增材制造工程技术研究中心 青岛 266520)
}

\begin{abstract}
摘要: 针对当前聚合物基复合材料(Polymer matrix composites, PMC)成型存在打印分辨率低、打印材料受限、成型结构较为 简单、工序复杂等方面的不足和局限性, 尤其是还面临难以实现宏/微结构跨尺度高效制造的挑战性难题, 提出一种基于电场 驱动熔融喷射 PMC 高分辨率 $3 \mathrm{D}$ 打印新工艺。阐述了基于电场驱动熔融喷射 PMC 高分辨率 $3 \mathrm{D}$ 打印的基本原理和工艺流程。 通过试验, 揭示了主要工艺参数(碳填料含量、施加电压、螺杆转速、打印速度、加热温度等)对于打印件分辨率(精度)和质量 的影响及其规律。利用自主搭建的试验平台, 并结合试验优化的工艺参数和提出的两种打印模式, 实现了多层石墨烯/聚乳酸 (Polylactic acid,PLA)和多壁碳纳米管/PLA 复合材料微尺度三维网格、多层石墨烯/PLA 大高宽比薄壁圆环、多壁碳纳米管/PLA 复合材料柔性导电网格以及其他聚合物复合材料 3D 结构典型工程案例的制造。研究结果表明, 提出的电场驱动熔融喷射 $3 \mathrm{D}$ 打印能实现高分辨聚合物基复合材料成型(使用内径 $300 \mu \mathrm{m}$ 喷嘴, 实现了分辨率为 $40 \mu \mathrm{m}$ 的 PMC 特征结构制造), 而且还具 有大面积宏/微结构跨尺度集成制造的优势。
\end{abstract}

关键词：电场驱动熔融喷射；聚合物基复合材料；高分辨 3D 打印；跨尺度制造

中图分类号: TB332

\section{High-resolution 3D Printing of Polymer Matrix Composites Based on Electric-field-driven Fusion Jetting}

\author{
YANG Kun ZHANG Guangming LI Xiaoqiang YANG Jianjun \\ PENG Zilong LAN Hongbo
}

(Shandong Engineering Research Center for Additive Manufacturing, Qingdao University of Technology,

Qingdao 266520)

\begin{abstract}
There are many disadvantages for existing forming methods of polymer matrix composites which involve the low resolution, limited printed materials, and poor capability of making complex structures, tedious processes, etc. In particular, it is always a challenge issue to implement multi-scale fabrication for macro/micro structures with high effectiveness. A high-resolution 3D printing process of polymer matrix composites based on electric-field-driven fusion jet is proposed. The fundamental principle and process flow of the proposed method are described. Furthermore, the effects and rules of main process parameters (mass fraction of carbon filler, applied voltage, screw speed, printing speed, heating temperature, etc.) on the resolution (accuracy) and quality of printed parts are revealed by a series of experiments. Finally, using the experimental setup independently developed by the research group, combining with the optimized process parameters and two printing modes proposed, some typical cases are demonstrated which include the micro 3D grids of multi-layer graphene (MLG)/polylactic acid (PLA) and multi-walled carbon nanotubes (MWCNT)/PLA composites, the large-aspect-ratio thin-walled ring of MLG/PLA composite, and flexible conductive structures of MWCNT/PLA composite, as well as some PMC 3D functional parts. The results show that the new 3D printing process can
\end{abstract}

* 国家自然科学基金(51775288, 51805287)和山东省重点研发计划 (2018GGX103022)资助项目。20200117 收到初稿, 20200916 收到修改稿 
implement the high resolution printing for various PMS (using the nozzle with an inner diameter of $300 \mu \mathrm{m}$, the feature size of $40 \mu \mathrm{m}$ for PMC has been achieved), and the multi-scale manufacturing of macro/micro-structures made of MWCNT, MLG, short carbon fiber reinforcement material and PLA matrix material can be realized.

Key words: electric-field-driven fusion jetting; polymer matrix composites; high-resolution 3D printing; multi-scale fabrication

\section{0 前言}

聚合物基复合材料(Polymer matrix composites, $\mathrm{PMC})$ ，是指以有机聚合物为基体，纤维、颗粒或纳 米填料等为增强相的复合材料。不同于纯聚合物材 料, PMC 具有优良的力学性能(比强度大、高比模 量)、物理性能(阻燃、耐磨、抗疲劳、轻质)、热学 性能(热导率高)、电学性能(导电、电磁屏蔽)和磁学 性能等突出的优势 ${ }^{[1-2]}$, 尤其是近年出现的聚合物基 纳米复合材料 ${ }^{[1,3]}$, 通过添加各种纳米材料, 诸如一 维纳米材料(碳纳米管、纳米线、纳米纤维等)、二 维纳米(如石墨烯等)、三维纳米(纳米球、纳米晶粒), 具有更加卓越综合性能。此外, PMC 还具有结构、 性能的可设计性和可裁剪性的特点。PMC 目前已经 被应用于航空航天、汽车、生物医疗和组织工程(组 织支架、神经导管、假肢、肿瘤检测等)、电子、新 材料、能源(超级电容器、微型燃料电池、太阳能等)、 可穿戴设备、柔性传感器、机器人等诸多领域 ${ }^{[4-8]}$, 展示出巨大和广阔的工业化应用前景。

PMC 的成型工艺目前主要包括: 手糊成型、模 压成型、袋压成型、热压罐成型、层压或卷制成型、 纤维铺放 (缠绕)成型、喷射成型、浇铸成型等。然 而, 这些传统的 PMC 成型工艺存在成型结构简单、 工艺柔性和适用性差(较为复杂的结构都需要首先 制造模具)、生产成本高、制造周期长和效率低的问 题, 尤其是难以实现复杂三维结构和高精度(高分辨 率)结构产品的成型, 限制了在许多领域应用和普 及。近年发展起来的 $3 \mathrm{D}$ 打印技术为 PMC 的制备和 成型提供了一种全新的解决方案。

国内外研究人员已经提出多种基于 3D 打印 PMC 的成型工艺, 主要包括: 熔融沉积成型(Fused deposition modeling, FDM)、墨水直写(Direct ink writing, DIW)、 立体光固化成型(Stereolithography apparatus, SLA)和数 字光处理成型(Digital light processing, DLP)、选区激 光烧结(Selected laser sintering, SLS) 以及静电纺丝 (Electrospinning)等 ${ }^{[1,3-10]}$ 。但是现有的这些 PMC 3D 打 印工艺, 大多难以实现 PMC 低成本高分辨率成型, 尤 其还面临打印材料种类受限和普适性差(只适合特 定打印材料、具体材料形状和一定尺寸范围等)、材
料混合不均匀和无法实现宏/微结构跨尺度制造的 挑战性难题。

本文提出一种基于电场驱动熔融喷射 PMC 高 分辨率 3D 打印新工艺, 通过结合微型螺杆混合摚 拌、电场驱动熔融喷射、四级加热、挤出成形和喷射 成形两种打印模式等多种策略和方法, 实现 PMC 结 构的高分辨率打印以及宏/微结构跨尺度高效制造。

\section{1 基本原理和工艺流程}

\section{1 基本原理}

基于电场驱动熔融喷射 PMC 高分辨率 3D 打印 是一种结合螺杆主动混料和电场驱动熔融喷射沉积 的新型复合材料高分辨成型工艺。该系统主要包括 打印喷头模块、三轴运动模块、高压电源模块、打 印基底等，其中，打印喷头模块由微型螺杆、驱动 电机、机筒、四级加热器、导电喷嘴组成, 高压电 源与导电喷嘴连接, 如图 1a 所示。基于电场驱动熔 融喷射 PMC 高分辨率 3D 打印的基本原理: 首先, 对组分材料(固体粉末/颗粒)进行充分均匀的预混; 然后, 利用打印喷头螺杆的剪切、挤压等综合作用, 使增强材料均匀分布在熔融状态的基体材料中，形 成均相复合材料; 随后, 熔融态均相复合材料经过 螺杆稳定输送挤出到达喷嘴末端(喷嘴内径 100 $500 \mu \mathrm{m})$, 喷嘴内的熔滴在背压的作用下被挤出喷嘴 并在表面张力作用下形成半球状(弯液面); 在导电 喷嘴施加正的高电压，处于喷嘴下方的打印基底被 极化, 基底的电荷发生重新分布(负电荷被吸引到基 底上表面，正电荷被排斥到基底下表面)，基底上表 面的负电荷与连接正极的导电喷嘴形成并建立静电 场。在电场作用小, 被挤出的熔滴弯液面在电场力、 表面张力、重力、黏滞力等综合作用下逐渐被拉伸 变长形成泰勒雉(图 1c), 当静电场力超过表面张力, 泰勒雉尖端喷射形成连续稳定雉射流, 喷射沉积到 基底并冷却固化成型(微尺度/亚微尺度线结构), 结 合喷头/打印平台三轴运动, 实现 PMC 高分辨率 $3 \mathrm{D}$ 结构成型。

同时考虑实际打印需要, 兼顾打印效率和打印 精度, 以实现大面积宏/微结构跨尺度的 3D 打印, 解决大尺寸高精度零件的制造难题。提出两种 $3 \mathrm{D}$ 
打印工作模式: 挤出成形和喷射成形, 如图 $1 \mathrm{~b}$ 所示。 其中, 挤出成形模式主要利用螺杆对熔融材料的挤 压作用, 将材料 “推/挤出” 喷嘴并沉积到基底, 其 打印特征尺寸取决于喷嘴直径, 可用于实现宏尺度 结构快速打印; 喷射成形模式利用电场力将材料“拉 出” 喷嘴, 利用泰勒雉 “缩颈效应”, 实现微尺度特 征结构打印。

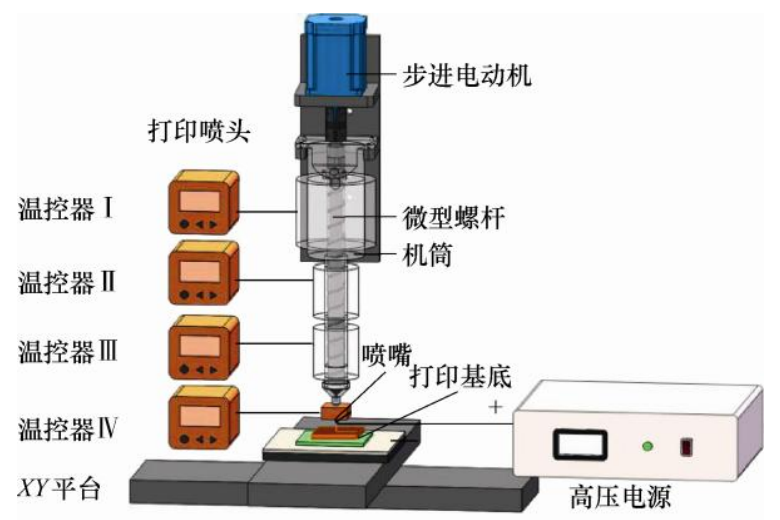

(a) 系统总体结构

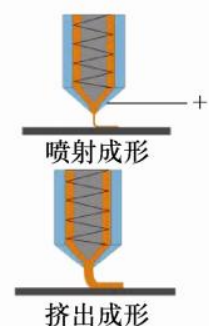

(b) 两种打印模式

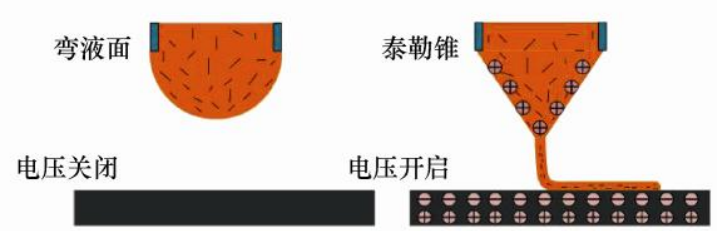

(c) 泰勒锥演变过程

图 1 电场驱动熔融喷射 PMC 高分辨率 3D 打印

提出的 3D 打印新工艺与传统的电场驱动熔融 喷射沉积技术打印纯聚合物相比, 由于增强相材料 (石墨烯、碳纳米管)的加入在不同程度上影响聚合 物的导电性能, 且增大了 PMC 黏度。因此, 打印 过程中, 高压电源可能引起材料与设备导电连通短 路、粘度过大导致混料和挤出困难等问题, 都增大 了实现高填充量复合材料的打印难度。

为了确保复合材料能够被均匀混合和稳定输送 挤出, 基于 PMC 在单螺杆中主要经历 “固态一高 弹态一黏流态” 三个过程, 分别对应螺杆的加料段、 压缩段和计量段, 在螺杆外壁设置四级加热器实现 温度分区精准调控。具体来讲: 以基体塑料的熔点
$\left(T_{\mathrm{m}}\right)$ 为依据进行温度划分, 根据前期试验结果, 在 第一加热器中, 物料在加料段初步软化, 温度设定 为 $(0.73 \sim 0.88) T_{\mathrm{m}}$; 在第二加热器中, 物料在压缩段 受螺杆剪切力与加热器传热共同作用被压缩和塑 化, 通常该段温度要高于加料段温度, 常取 $(0.76$ $0.97) T_{\mathrm{m}}$; 在第三加热器中, 物料在计量段被进一步 塑化, 使黏流态的物料趋于均匀化, 通常该段温度 一般等于或略高于压缩段温度，该段对混合材料的 质量起决定性作用, 常取 $(0.77 \sim 1.00) T_{\mathrm{m}}$; 在第四加 热器中, 物料在喷嘴内处于均匀塑化的状态, 为保 证材料流动性, 通常该段温度较机筒温度要高, 常 取 $(0.79 \sim 1.03) T_{\mathrm{m}}$ 。

\section{2 工艺流程}

基于电场驱动熔融喷射 $\mathrm{PMC}$ 高分辨率 $3 \mathrm{D}$ 打印 具体流程如下。

(1) 打印数据文件准备和打印模式确定。(1) 使 用建模软件绘制三维模型, 将模型文件(STL)导入切 片软件中; (2) 根据三维模型结构特征和精度要求, 选择打印模式。当要求精度较高时则选择喷射模式, 当要求高效率, 则选择挤出模式, 当部分结构要求 高精度, 部分结构精度要求较低时, 可在打印时进 行两种模式切换; (3) 设置打印参数(层厚、填充等 参数), 生成打印路径 $\mathrm{G}$ 代码, 并将生成的 $\mathrm{G}$ 代码 文件导入工控机以备打印。

（2）打印预处理。(1) 备料，以热塑性聚合物粉 末( $\mathrm{A}$ 料)+改性粉末 (B 料)共混为例, 将 $\mathrm{A}$ 材料和 $\mathrm{B}$ 材料按配比(重量比或体积比)完成预混合; (2) 设备 使能。开启工控机, 加载打印 $\mathrm{G}$ 代码, 设置打印速 度, 调整打印喷头与基底距离, 设置打印原点, 开 启调速器, 设置螺杆转速, 开启各温控器, 并达到 设定温度; (3) $Z$ 轴上升设定高度值(例如 $10 \mathrm{~mm}$ ), 待机打印。

（3）打印结构件。打印喷头和打印床移动到打 印工位, 螺杆按设定转速转动的基础上, 完成步骤 (1) 送料, 将混合好的复合材料放置到进料装置中, 材料在自身重力或者施加外力的作用下向下运动; (2) 混料, 复合材料在进料口处初步受热软化, 在螺 杆的螺旋挤压作用下, 继续向下运动, 处在单螺杆 与筒壁间隙处的材料受剪切、挤压作用完成混合和 输送, 混合均匀的复合材料最终被输送至导电喷嘴; (3) 排料, 持续加料, 直至出料稳定; (4) 打印结构 件, 运行 $\mathrm{G}$ 代码, 打印结构件, 如果是挤出成形, 利用打印喷头的单螺杆挤出打印材料; 如果是喷射 成形，开启高压电源，喷射打印材料。

(4) 打印后处理。打印完成, 关闭高压电源、 
调速器, 取下打印完成的复合材料结构件, 并根据 需要进行相应的后处理等工作诸如去除支撑、表面 光整等。

\section{2 试验装置和材料}

\section{1 试验装置}

自主搭建试验平台如图 2 所示。它主要包括: 打印喷头模块、三轴运动模块、高压电源模块、温 控模块、打印基底、控制模块等。其中，打印喷头 安装在 $Z$ 轴工作台上, 随 $Z$ 轴完成 $Z$ 向移动, 打印 基底固定在 $X Y$ 模组工作台上, 随 $X Y$ 轴完成 $X 、 Y$ 向移动, 高压电源通过导线与打印喷头的导电喷嘴 相连接, 提供打印所需电场力, 各部分在控制模块 的综合调控下, 协调动作。上述控制模块包括螺杆 转速控制单元、三轴运动控制单元、温度控制单元、 其他辅助协同控制单元，各个控制单元协同工作。

打印喷头为微型单螺杆摚拌挤出装置, 主要包 括微型螺杆和机筒两部分。本设计采用槽深渐变型 等距不等深形式螺杆, 按照目前流行的三段式, 加 料段、压缩段、计量段进行分段设计。此外, 为了 防止加电时, 金属机筒与导电喷嘴导通而影响装置 其它电子器件工作, 将机筒分为两部分设计, 一部 分采用金属材料, 一部分采用绝缘材料, 绝缘材料 一端连接金属机筒, 一端连接金属机头, 各段通过 螺纹实现密封连接, 绝缘材料将连接高压电源的喷 嘴与机筒隔离, 避免设备连电短路。所用绝缘材料 为耐高温聚醚醚酮(Polyetheretherketone, PEEK)。

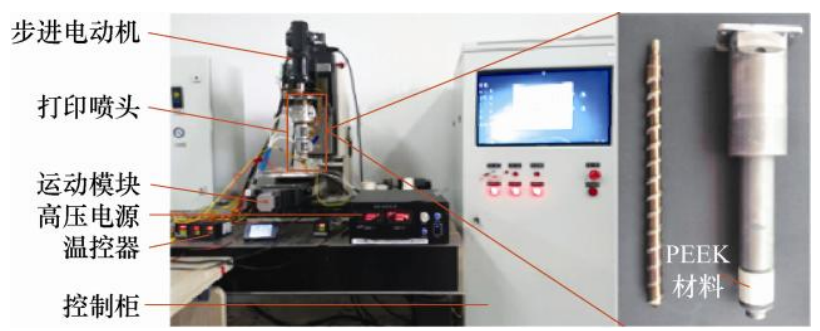

图 2 搭建试验装置照片

\section{2 试验材料}

基体材料: 聚乳酸(Polylactic acid, PLA), 使用 Nature Works PLA-4032D 粉末, 粒径 $58 \mu \mathrm{m}$, 东莞 市丹盛塑胶有限公司。

改性材料: (1) 多壁碳纳米管(Multi-walled carbon nanotube, MWCNT), 使用工业级 MWCNT 粉末, 纤 维内径 3 5 nm、纤维外径 8 15 $\mathrm{nm}$ 、纤维长度 3 $12 \mu \mathrm{m}$; (2) 多层石墨烯(Multi-layer graphene, MLG), 使用工业级 MLG 粉末, 片层直径 10 50 $\mu \mathrm{m}$ 、片层厚
度 3.4 7.0 nm，苏州碳丰石墨烯科技有限公司。

增强材料：短切碳纤维 (Short carbon fiber, $\mathrm{SCF}$ ), 使用 SCF 粉末, 其纤维直径 $7.5 \mu \mathrm{m}$, 纤维长 度 $100 \mu \mathrm{m}$ ，复材易购(北京)科技有限公司。

\section{3 试验结果与讨论}

影响碳填料/PLA 复合材料打印质量和精度的 主要因素包括: 碳填料含量、施加电压、螺杆转速、 打印速度、加热温度等。而且这些因素相互作用相 互影响, 理论上存在一个最优的打印工艺窗口, 采 用最优工艺参数打印, 能获得比较理想的打印质量 和精度。本文使用 MWCNT、MLG 以及 SCF 等对 其分散效果进行验证, 随后使用 MWCNT/PLA 复合 材料研究揭示主要工艺参数对打印质量的影响 规律。

\section{1 复合材料的分散效果}

为了验证打印复合材料的均匀分散能力, 分别 制备了不同填充材料(MWCNT、MLG 以及 SCF 等) 的复合材料, 并对其断面的微观形貌进行观察。如 图 3 所示, 三种碳填料在基体中的分布较为均匀, 且随着碳填料含量(质量分数)的增加, 纤维分布越 来越密集, 材料内部微观界面越来越多; 另外, 相 较于 MLG 来说, MWCNT 在基体材料中的分散更 均匀, 更密集; SCF 的分布和打印方向同向, 表明 SCF 在剪切场和拉伸场的作用下对齐，并沿熔体流 动方向取向 ${ }^{[12-13]}$, 这对复合材料的力学性能有很大 影响。通过以上结果可知, 本装置具备均匀分散增 强相的能力, 可用于 PMC 的制备与结构一体化 打印。

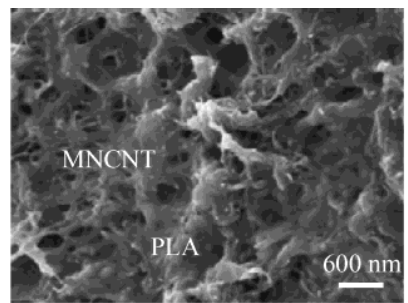

(a) $2 \% \mathrm{MWCNT} / \mathrm{PLA}$

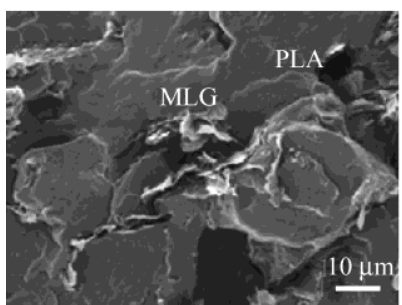

(c) $2 \% \mathrm{MLG} / \mathrm{PLA}$

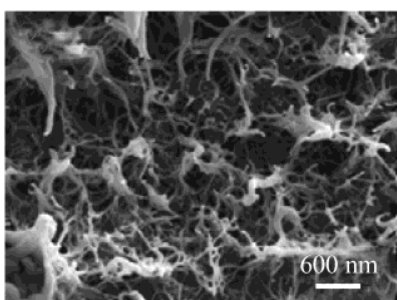

(b) $9 \% \mathrm{MWCNT} / \mathrm{PLA}$

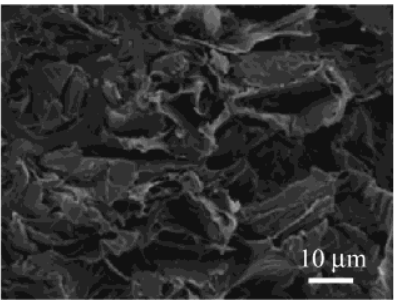

(d) $20 \% \mathrm{MLG} / \mathrm{PLA}$ 


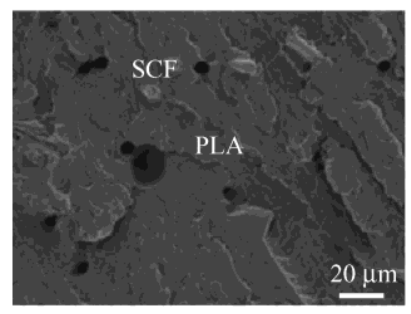

(e) $2 \% \mathrm{SCF} / \mathrm{PLA}$

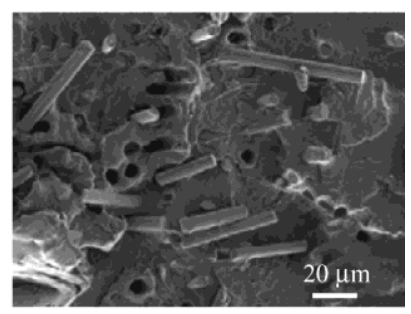

(f) $10 \% \mathrm{SCF} / \mathrm{PLA}$
图 3 打印复合材料断面微观形貌的 SEM 图像

\section{2 基于稳定出料的填料含量和加热温度匹配}

MWCNT/PLA 复合材料的黏度(流动性)主要受 加热温度和 MWCNT 含量的影响。考虑到 MWCNT 含量对黏度的影响, 本试验主要研究了不同 MWCNT 含量的复合材料在出料速度相同的前提 下, 各物料段, 包括加料段、压缩段、计量段和喷 嘴所使用温度值的变化。其结果如图 4 所示, 出料 速度为 $13 \mathrm{~mm}^{3} / \mathrm{s}$ 时, 不同 MWCNT 含量的复合材 料所使用加热温度的大小关系为: $0.5 \%<0 \%<1 \%<2 \%$ 。这主要归因于 MWCNT 提高了 复合材料的导热性和黏度。在 PLA 中添加少量 MWCNT(质量分数低于 $1 \%$ ), 复合材料的黏度变化 不大, 但其热导率大大提高, 而复合材料的黏度会 随温度升高而降低, 物料在料筒内流动性变好, 为 保证相同的出料速度, 需要降低外部施加温度; 随 着 MWCNT 含量的增加(质量分数大于等于 $1 \%$ ), 复 合材料的黏度大大增加, 热导率的提高不足以改善 材料的流动性, 为保证相同的出料速度, 需要提高 外部施加温度。

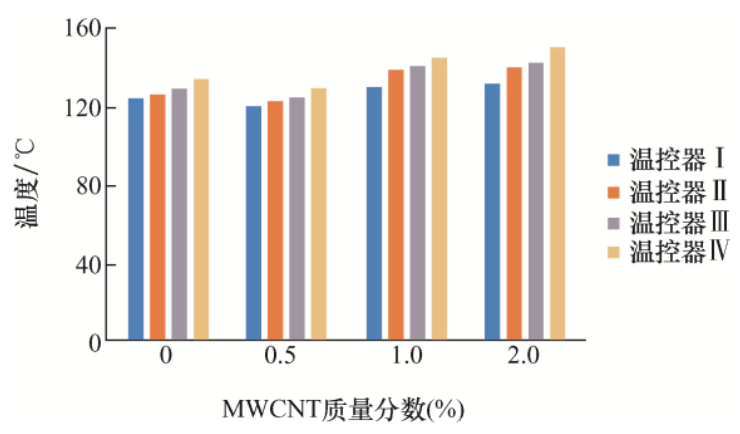

图 4 不同 MWCNT 含量的复合材料所使用 加热温度的柱状图

\section{3 工艺参数对线宽的影响}

以 $1 \% \mathrm{MWCNT/PLA}$ 复合材料为例, 在固定加 热温度和打印高度的前提下, 分别研究了电压、螺 杆转速和打印速度对打印线宽的影响, 通过以下系 列试验研究, 揭示主要工艺参数对于打印过程的影 响及其规律, 并优化出适合的打印工艺参数。试验 固定工艺参数为: 内径 $300 \mu \mathrm{m}$ 武藏式喷嘴, 加料
段温度 $122{ }^{\circ} \mathrm{C}$, 压缩段温度 $123{ }^{\circ} \mathrm{C}$, 计量段温度 $124{ }^{\circ} \mathrm{C}$, 喷嘴温度 $125{ }^{\circ} \mathrm{C}$, 打印高度 $500 \mu \mathrm{m}$ 。

(1) 电压对线宽的影响。

固定螺杆转速和打印速度, 试验研究了在实 现稳定喷射的条件下, 线宽随电压的变化规律。 工艺参数为: 螺杆转速 $3 \mathrm{r} / \mathrm{min}$, 打印速度 $10 \mathrm{~mm} / \mathrm{s}$, 电压 $1000 \sim 1900 \mathrm{~V}$, 取值间隔为 $150 \mathrm{~V}$ 。其结果如 图 5 所示, 电压在 $1000 \sim 1450 \mathrm{~V}$ 区间, 线宽随电 压的增加而增大，而在 1450 1900 V 区间，线宽 则趋于稳定。分析其原因: 电压增大, 尖端极化电 荷量增加, 电场变强, 弯液面尖端受到的电场力变 大, 在强电场的作用下, 突破尖端的熔滴量增加, 射流变粗，线宽逐渐增大。但受制于 $1 \%$ MWCNT/PLA 复合材料的极化电荷量, 突破尖端的 熔滴量达到极限, 射流粗细基本不变, 线宽趋于稳 定。但整体来看, 线宽受电压的影响较小, 其值在 $60 \sim 90 \mu \mathrm{m}$ 之间。

(2) 螺杆转速对线宽的影响。

固定施加电压和打印速度, 试验研究了在实 现稳定喷射的前提下，线宽随螺杆转速的变化规 律。试验采用工艺参数为: 电压 $1200 \mathrm{~V}$, 打印速 度 $15 \mathrm{~mm} / \mathrm{s}$, 螺杆转速 $3 \sim 15 \mathrm{r} / \mathrm{min}$, 取值间隔为 $2 \mathrm{r} / \mathrm{min}$ 。螺杆转速对于打印线宽的影响和规律如 图 6 所示。

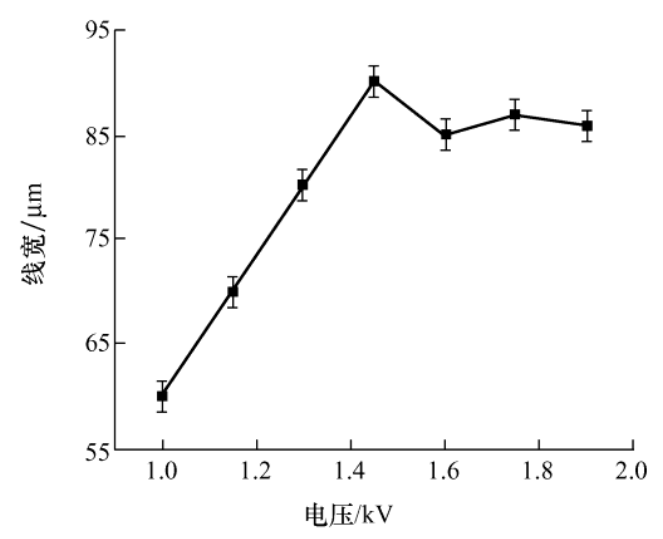

图 5 电压对线宽的影响

随着螺杆转速的增加, 线宽逐渐增加, 且增 长幅度较快。这种现象可能是由于两方面的原因, 一方面, 根据 BAGHERIASL 等 ${ }^{[14]}$ 建立的纳米微 晶纤维素和 PLA 复合材料的剪切流变特性, 螺杆 转速越大, 料筒内材料所受剪切力越大, 材料受 强剪切而变稀; 另一方面, 螺杆转速增加, 材料 输送速度变快。因此, 随着螺杆转速的增大, 材 料黏度降低, 熔体流量增大, 导致射流变粗, 从 而线宽增加。 


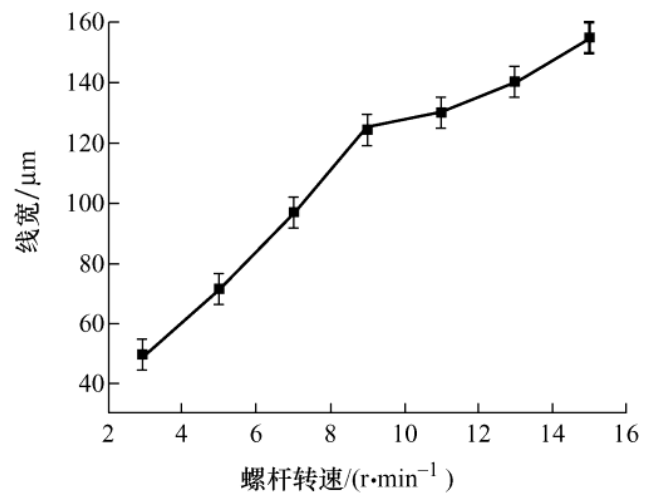

图 6 螺杆转速对线宽的影响

（3）打印速度对线宽的影响。

固定施加电压和螺杆转速, 试验研究了在实现 稳定喷射的前提下，线宽随打印速度的变化规律。 试验采用工艺参数为: 电压 $1300 \mathrm{~V}$, 螺杆转速 $3 \mathrm{r} / \mathrm{min}$, 打印速度 $5 \sim 35 \mathrm{~mm} / \mathrm{s}$, 取值间隔为 $5 \mathrm{~mm} / \mathrm{s}$ 。 打印速度对于打印线宽的影响和规律如图 7 所示: 随着打印速度增加, 线宽变细, 且线宽呈指数曲线 减小。分析其原因：当射流沉积到基底上之后，射 流与基底之间粘连, 随着打印平台的移动, 在粘性 拖拽力的作用下, 泰勒雉受力发生形变, 并将射流 拉伸变细, 且平台运动速度越快, 射流拉伸效果越 强，线宽也就越细。

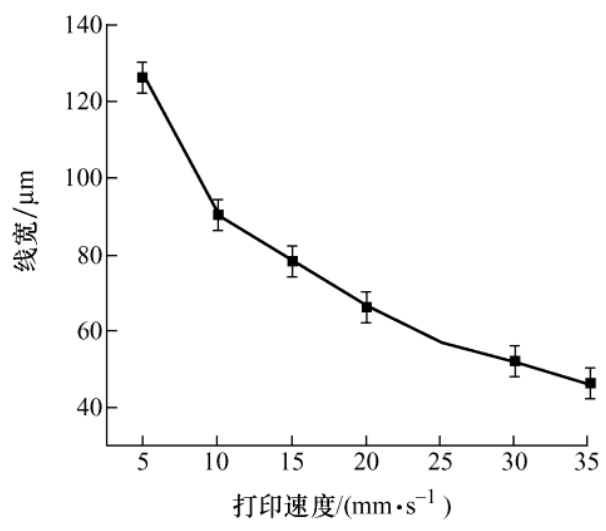

图 7 打印速度对线宽的影响

\section{4 典型案例}

碳纳米管、石墨烯以及碳纤维的聚合物复合 材料在组织支架、神经导管、柔性/可穿戴应变传 感器、可植入电子、微能源等诸多领域有着巨大 的需求。因此, 通过电场驱动熔融喷射聚合物基 复合材料高分辨率 $3 \mathrm{D}$ 打印以及优化的工艺参数, 并结合两种打印模式, 打印了 MWCNT、MLG 以 及 $\mathrm{SCF}$ 等多种 PMC 结构, 以此展示提出方法的
可行性和有效性。喷嘴选用内径为 $300 \mu \mathrm{m}$ 武藏 式喷嘴, 采用喷射模式(线宽小于 $150 \mu \mathrm{m}$ ) 打印了 微尺度三维网格大高宽比薄壁圆环; 采用挤出模 式(线宽 $150 \mu \mathrm{m}$ 以上)打印了柔性导电网格和宏观 结构件。

\section{1 微尺度三维网格}

图 8 为使用喷射成形模式打印的 $1 \%$ MWCNT/PLA 复合材料三维网格结构。打印工艺参 数: 打印高度为 $500 \mu \mathrm{m}$, 喷嘴温度为 $125^{\circ} \mathrm{C}$, 电压 为 $1000 \mathrm{~V}$, 螺杆转速为 $3 \mathrm{r} / \mathrm{min}$, 打印速度为 $10 \mathrm{~mm} / \mathrm{s}$ 。如图 8a 所示, 网格面积为 $40 \mathrm{~mm} \times 40 \mathrm{~mm}$, 从左到右单元网格的周期依次减小。图 $8 \mathrm{~b}$ 为局部放 大 SEM 图, 线宽为 $100 \mu \mathrm{m}$, 周期 $600 \mu \mathrm{m}$ 左右, 其 线宽为 $120 \mu \mathrm{m}$, 孔径为 $350 \mu \mathrm{m}$ 。同时, 相比较纯 PLA 结构, MWCNT 的加入会使打印线条的表面 变得粗䊁, 粗粘表面可以增加细胞黏附点, 有助 于增加细胞密度。因此, 使用本技术通过控制打 印周期、打印线宽和打印路径，可以用于所需孔 径、孔隙率以及孔形状的复合材料生物支架的灵 活和高效制备。加之，具有导电特性的 MWCNT/PLA 复合材料支架和电刺激结合还可以 显著提高脊髓损伤治疗中神经元干细胞定向分化 和轴突定向生长 ${ }^{[15]}$ 。

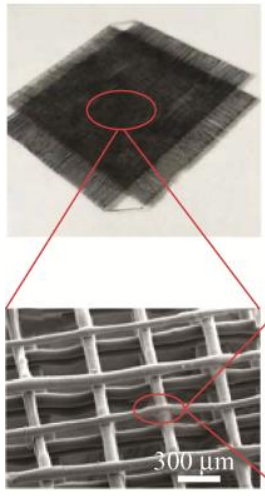

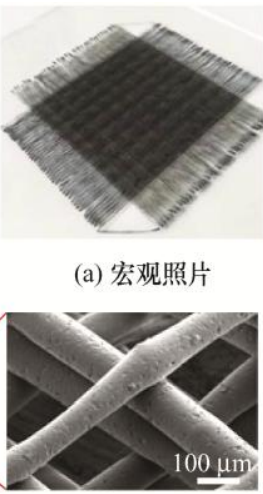

(b) SEM照片

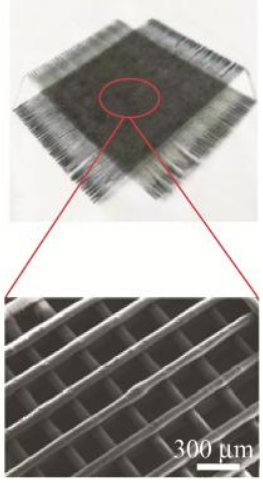

300 un
图 $81 \%$ MWCNT/PLA 复合材料的 6 层三维网格结构

\section{2 大高宽比薄壁圆环}

具有大高宽比的薄壁结构一直是高精度 3D 打 印的一个难点, 为了展示该技术的大高比成型能力, 使用喷射成形模式打印了 MLG/PLA 复合材料薄壁 圆环结构。试验打印参数为: 螺杆转速 $1 \mathrm{r} / \mathrm{min}$, 其 余打印参数见表 1。其中, 图 9a 是直径渐变圆环, 其下底直径 $10 \mathrm{~mm}$, 上底直径 $8 \mathrm{~mm}$, 壁厚 $100 \mu \mathrm{m}$, 总体高度 $3 \mathrm{~mm}$, 即雉度为 $2: 3$; 图 $9 \mathrm{~b}$ 是直筒圆环, 其直径 $20 \mathrm{~mm}$, 壁厚 $70 \mu \mathrm{m}$, 总体高度 $2 \mathrm{~mm}$; 图 
$9 \mathrm{c}$ 是直筒圆环, 其直径 $30 \mathrm{~mm}$, 壁厚 $120 \mu \mathrm{m}$, 总 体高度 $2 \mathrm{~mm}$ 。

表 1 MLG/PLA 复合材料薄壁圆环打印参数

\begin{tabular}{ccccc}
\hline $\begin{array}{c}\text { MLG 质量 } \\
\text { 分数 }(\%)\end{array}$ & $\begin{array}{c}\text { 喷嘴温度 } \\
/{ }^{\circ} \mathrm{C}\end{array}$ & $\begin{array}{c}\text { 施加电压 } \\
/ \mathrm{V}\end{array}$ & $\begin{array}{c}\text { 打印高度 } \\
/ \mu \mathrm{m}\end{array}$ & $\begin{array}{c}\text { 打印速度 } \\
/\left(\mathrm{mm} \cdot \mathrm{s}^{-1}\right)\end{array}$ \\
\hline 0.5 & 130 & 3000 & 500 & 6 \\
1 & 135 & 2900 & 400 & 7 \\
\hline
\end{tabular}

图 10 是 $0.5 \% \mathrm{MLG} / \mathrm{PLA}$ 复合材料薄壁圆环电 镜图, 从内圈、外圈以及断面三个角度观察薄壁圆 环, 可以看出, 打印结构具有较大的深宽比, 层与 层之间逐渐错开, 形成直径渐变圆环。由此展现了 电场驱动熔融喷射沉积技术具有高分辨率、高精度 的制造能力。

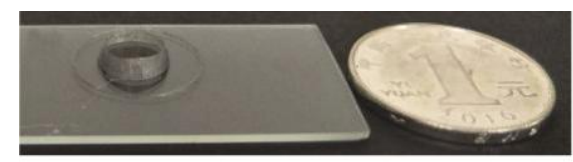

(a) $0.5 \% \mathrm{MLG} / \mathrm{PLA}$ 直径渐变圆环

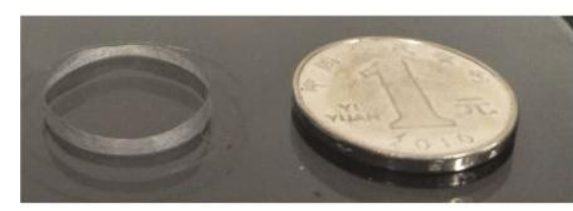

(b) $0.5 \% \mathrm{MLG} / \mathrm{PLA}$ 直筒圆环

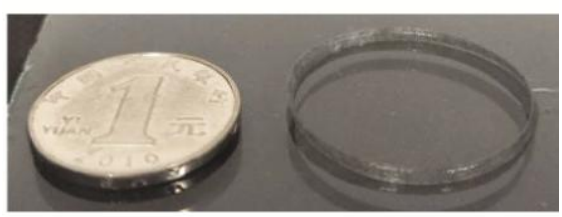

(c) $1 \% \mathrm{MLG} / \mathrm{PLA}$ 直筒圆环

图 9 MLG/PLA 复合材料薄壁圆环
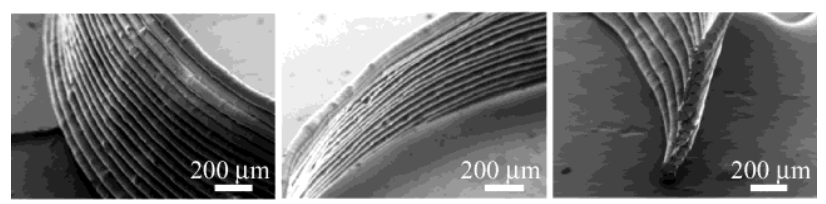

图 $10 \quad 0.5 \%$ MLG/PLA 复合材料薄壁圆环微观 形貌的 SEM 图像

\section{3 柔性导电网格}

图 11 为使用挤出成形模式制备的 MWCNT 含 量分别为 $2 \% 、 5 \% 、 7 \% 、 9 \%$ 的 MWCNT/PLA 复合 材料各向异性柔性网格结构, 其尺寸为 $60 \mathrm{~mm} \times$ $60 \mathrm{~mm} \times 0.08 \mathrm{~mm}$, 试验打印参数见表 2 。该结构可 以实现不同程度的弯曲, 且在对角方向上拉伸性能 最好, 在其余方向几乎不能拉伸。这主要是因为正 交单元结构上的不同造成的, 通过设计弹性可变形 结构, 使之在该方向上可以自由运动, 在垂直于该
方向上不能运动 ${ }^{[13]}$ 。

表 2 MWCNT/PLA 复合材料各向异性柔性网格打印参数

\begin{tabular}{cccc}
\hline $\begin{array}{c}\text { MWCNT 含量 } \\
(\%)\end{array}$ & 喷嘴温度 ${ }^{\circ} \mathrm{C}$ & $\begin{array}{c}\text { 螺杆转速 } \\
/\left(\mathrm{r} \cdot \mathrm{min}^{-1)}\right.\end{array}$ & $\begin{array}{c}\text { 打印速度 } \\
/\left(\mathrm{mm} \cdot \mathrm{s}^{-1}\right)\end{array}$ \\
\hline 2 & 145 & 10 & 20 \\
5 & 155 & 8 & 15 \\
7 & 150 & 7 & 10 \\
9 & 160 & 10 & 10 \\
\hline
\end{tabular}

复合材料的导电能力通过电导率的大小来体 现, 通常来说, 电导率越大, 导电能力越好。本研 究采取的计算方式如下: (1) 从结构中截取一小段材 料, 并将截取的材料认为是一个长方体薄片, 其长 度记为 $L$, 截面积记为 $A$; (2) 用万用表测量两端电 阻, 其电阻值记为 $R$; (3) 由公式 $\rho=R A / L$ 和公式 $\sigma=1 / \rho$ 分别计算得到电阻率值以及电导率值, 最后 采用 MESTEK 公司生产的 DP3020 大功率直流稳压 电源, 使用电压范围为 $0 \sim 32 \mathrm{~V}$, 对制备样件进行 导电测试。
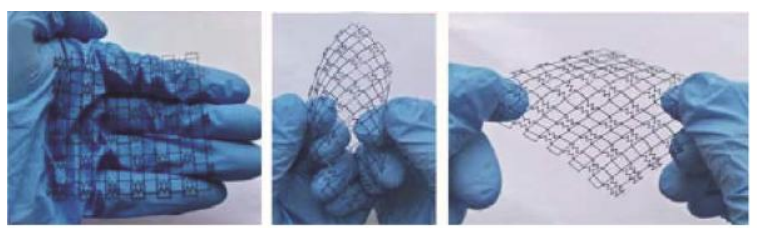

(a) 之字形
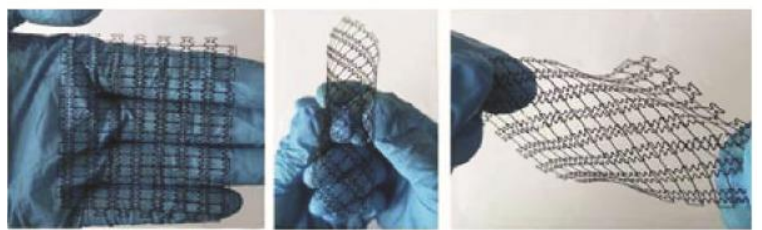

(b) 正弦形
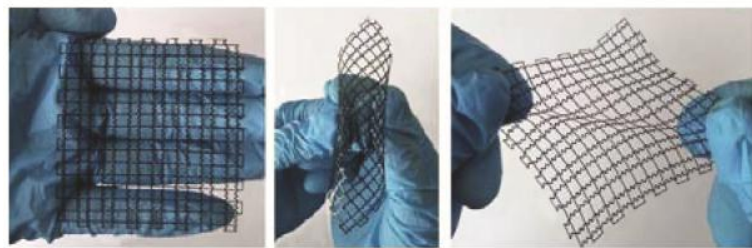

(c) 铪齿形

图 11 MWCNT/PLA 复合材料各向异性柔性导电网格

电导率和电阻率随 MWCNT 含量的变化规律 如图 $12 \mathrm{a}$ 所示。可以看出, 随着 MWCNT 含量的增 加, 复合材料的电导率变大, 电阻率减小, MWCNT 添加量的提升大大改善了复合材料的导电能力。将打 印的 MWCNT/PLA 复合材料各向异性柔性网格与发 光二极管连接, 并将其接到直流稳压电源上, 形成导 通回路, 连接方式具体见图 12b。以 $9 \%$ MWCNT/PLA 复合材料之字形柔性网格为例, 设置电压值为 $32 \mathrm{~V}$ (通 过试验, 电压范围在 $10 \sim 32 \mathrm{~V}$, 均能使二极管导通), 
开启电源后, 可以看到, 二极管导通变亮, 证明打印 的 MWCNT/PLA 复合材料在较小电压下便可以导通, 材料从绝缘材料完成了向导电材料的转变。

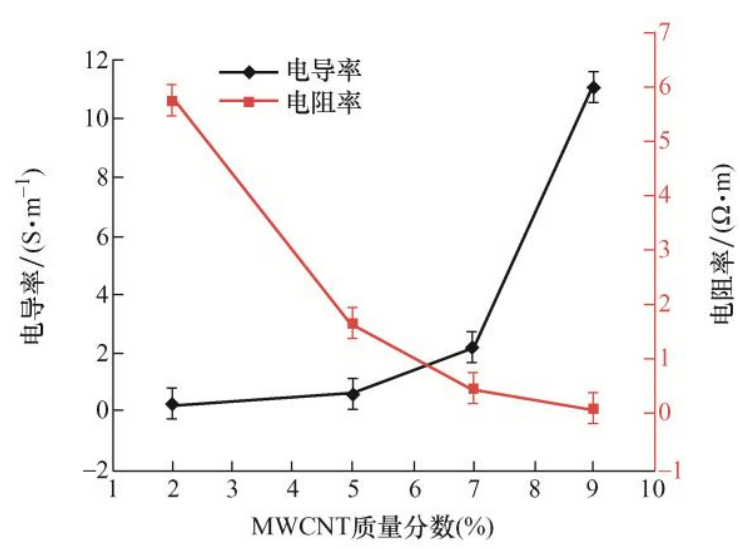

(a) 电阻率和电导率随MWCNT含量的变化规律图

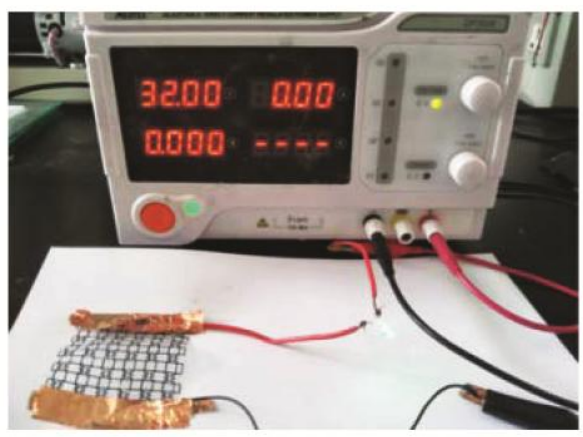

(b) 作为导电元件导通二极管

图 12 MWCNT/PLA 复合材料各向异性柔性网格

\section{4 复合材料宏尺度结构}

碳纤维是一种高强度、高模量的新型纤维材料, 其质量比金属铝材料还要轻, 但是强度却高于钢铁 材料, 同时还具备优良的耐热性、耐腐蚀性以及耐 疲劳性。因此, 将碳纤维与聚合物相结合, 能够制 造出更轻、更强的优异材料。图 13 为采用挤出模式 打印的 SCF/PLA 复合材料蜂窝状结构及其力学性 能。如图 13a 所示, 样件尺寸为 $60 \mathrm{~mm} \times 25 \mathrm{~mm} \times$ $3 \mathrm{~mm}$, 蜂窝填充率为 $40 \%$; 碳纤维质量分数为 $0 \%$, $2 \%, 10 \%$ 。图 13b 是不同碳纤维含量蜂窝结构的应 力-应变曲线图; 图 13c 是为拉伸强度和断裂伸长率 随碳纤维含量的变化规律图。由图 13 可以看出, 随 着碳纤维含量的增加, 复合材料的拉伸强度逐渐提 高, 但其断裂伸长率先提高后下降。其中, 与纯料 相比, 碳纤维含量为 $2 \%$ 时的拉伸强度由纯料的 35.8 $\mathrm{MPa}$ 提高到 $39.9 \mathrm{MPa}$, 提高了 $11 \%$; 碳纤维含量为 $10 \%$ 时的拉伸强度由纯料的 $35.8 \mathrm{MPa}$ 提高到 51.9 $\mathrm{MPa}$, 提高了 $45 \%$ 。另外, 断裂伸长率分别于由 $5.9 \%$ 提高到 $7.4 \%$ 以及由 $5.9 \%$ 下降到 $5.1 \%$ 。

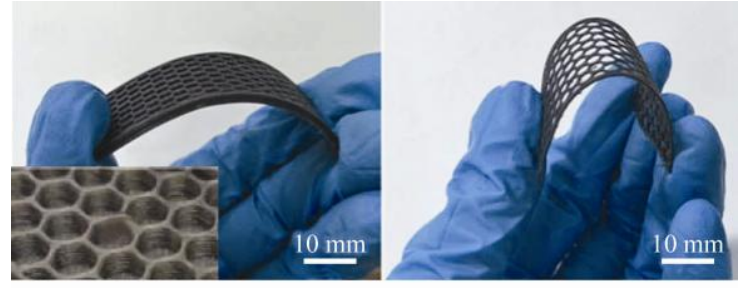

(a) 蜂窝状结构的宏观照片

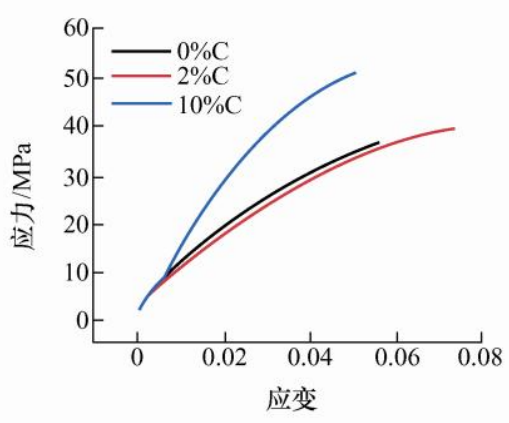

(b) 三种不同SCF含量的应力-应变曲线

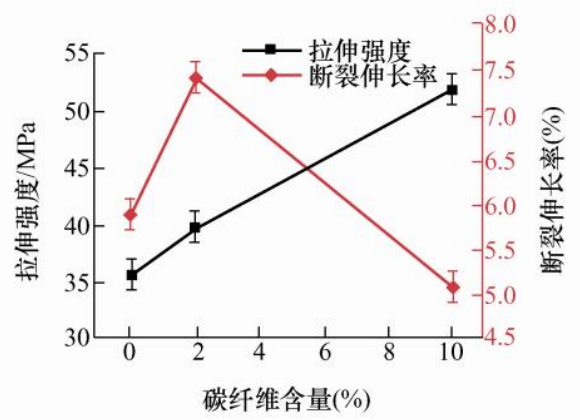

(c) SCF含量对拉伸强度和伸长率的影响规律

图 $13 \mathrm{SCF} / \mathrm{PLA}$ 复合材料蜂窝状结构及其力学性能

以外，为了进一步展示宏尺度 3D 结构的制造能 力, 我们使用挤出成形模式成功制备出, MWCNT/PLA 的法兰、齿轮以及半球结构, 氧化铝 $\left(\mathrm{Al}_{2} \mathrm{O}_{3}\right) / \mathrm{PLA}$ 的多 孔原片、碳化硅 $(\mathrm{SiC}) / \mathrm{PLA}$ 齿轮等结构。如图 14 所示, 打印零件具有较高的表面质量。使用本方法能够实现 多种零件结构的高效、低成本制造。
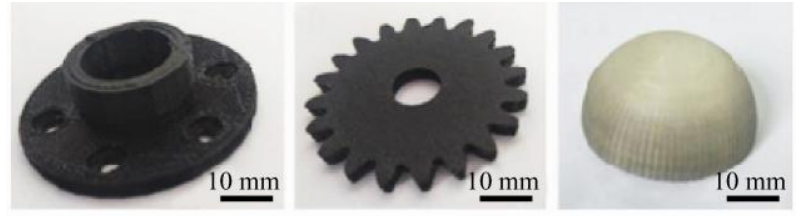

(a) MWCNT/PLA复合材料
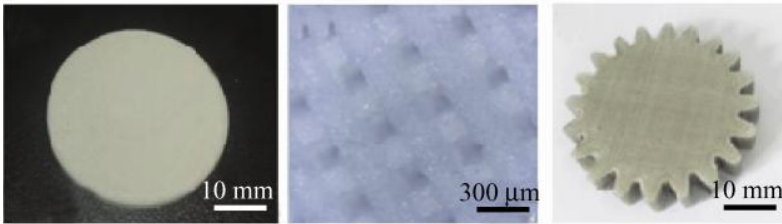

(b) $\mathrm{Al}_{2} \mathrm{O}_{3} /$ PLA复合材料

(c) SiC/PLA复合材料 


\section{5 结论}

(1) 提出的电场驱动熔融喷射 PMC 高分辨率 3D 打印使用微型螺杆作为混料装置, 实现了物料的 均匀混合和稳定输送; 根据物料在不同阶段不同的 热量需求, 设置四级加热系统, 实现温度分区精准 调控, 保证物料顺序均匀熔融和输送; 提出了挤出 和喷射沉积两种打印模式, 能够同时兼顾打印效率 和打印精度, 根据成型件特征结构的不同, 选用不 同的打印模式, 实现了大面积宏/微结构跨尺度集成 制造。

（2）揭示了主要工艺参数(碳填料含量、施加 电压、螺杆转速、打印速度、加热温度等)对于打 印件分辨率(精度)和质量的影响及其规律。线宽 随电压的增加先增大最后趋于稳定, 随螺杆转速 的增加而增大，随打印速度的增加而减小。打印 了质量分数为 $0 \sim 9 \%$ 多壁碳纳米管 $(\mathrm{MWCNT})$ 、 0 20\%多层石墨烯(MLG)和 $0 \sim 10 \%$ 短切碳纤维 (SCF) 的 PLA 复合材料, 碳填料在基体中分布均 匀, 表明该技术具备均匀分散高固含量增强相的 能力。通过调节工艺参数, 使用内径 $300 \mu \mathrm{m}$ 武 藏式喷嘴, 具有高分辨(最细 $40 \mu \mathrm{m}$ ) 以及多尺度 $(40 \sim 300 \mu \mathrm{m})$ 的打印能力。

(3) 结合试验优化的工艺参数和提出的两种 打印模式, 实现了微尺度 MWCNT/PLA 和 MLG/ PLA 复合材料三维网格以及大高宽比 MLG/PLA 复合材料薄壁圆环打印; 宏尺度 MWCNT/PLA 柔 性导电网格结构以及 MWCNT/PLA、SCF/PLA、 氧化铝/PLA 以及碳化硅/PLA 等复合材料零件的 打印。该技术为复合材料的高分辨率成型以及宏/ 微跨尺度高效制造提供了一种较为理想的解决方 案, 具有工艺适应性/柔性好, 打印材料广泛, 成 型精度高的优势。

\section{参 考 文 献}

[1] WANG X, JIANG M, ZHOU Z, et al. 3D printing of polymer matrix composites: A review and prospective[J]. Composites Part B, 2017, 110: 442-458.

[2] GUPTA S, TAI N H. Carbon materials and their composites for electromagnetic interference shielding effectiveness in X-band[J]. Carbon, 2019, 152: 159-187.

[3] HUSSAIN F, HOJJATI M, OKAMOTO $\mathrm{M}$, et al. Polymer-matrix nanocomposites, processing, manufacturing, and application: An overview[J]. Journal of Composite
Materials, 2006, 40(17): 1511-1575.

[4] BEKAS D G, HOU Y, LIU Y, et al. 3D printing to enable multifunctionality in polymer-based composites: A review [J]. Composites Part B: Engineering, 2019, 179: 107540.

[5] LEIGH S J, BRADLEY R J, PURSSELL C P, et al. A simple, low-cost conductive composite material for 3D printing of electronic sensors[J]. PloS ONE, 2012, 7(11): e49365.

[6] LEE S J, ZHU W, NOWICKI M, et al. 3D printing nano conductive multi-walled carbon nanotube scaffolds for nerve regeneration[J]. Journal of Neural Engineering, 2018, 15(1): 016018.

[7] BARILE C，CASAVOLA C，DE CILLIS F. Mechanical comparison of new composite materials for aerospace applications[J]. Composites Part B: Engineering, 2019, 162: $122-128$

[8] WEI Y, QIAO Y, JIANG G, et al. A wearable skin-like ultra-sensitive artificial graphene throat[J]. ACS Nano, 2019, 13(8): 8639-8647.

[9] GUPTA P, RAJPUT M, SINGLA N, et al. Electric field and current assisted alignment of CNT inside polymer matrix and its effects on electrical and mechanical properties[J]. Polymer, 2016， 89: 119-127.

[10] VALINO A D, DIZON J R, ESPERA JR A H, et al. Advances in 3D printing of thermoplastic polymer composites and nanocomposites[J]. Progress in Polymer Science, 2019, 98: 101162.

[11] LEWICKI J P, RODRIGUEZ J N, ZHU C, et al. 3D-printing of meso-structurally ordered carbon fiber/polymer composites with unprecedented orthotropic physical properties[J]. Scientific Reports, 2017, 7: 43401.

[12] TEKINALP H L, KUNC V, VELEZ-GARCIA G M, et al. Highly oriented carbon fiber-polymer composites via additive manufacturing[J]. Composites Science and Technology, 2014, 105: 144-150.

[13] PATTINSON S W, HUBER M E, KIM S, et al. Additive manufacturing of biomechanically tailored meshes for compliant wearable and implantable devices[J]. Advanced Functional Materials，2019，29(32): 1901815.

[14] BAGHERIASL D, CARREAU P J, RIEDL B , et al. Shear rheology of polylactide (PLA)-cellulose nanocrystal 
(CNC) nanocomposites[J]. Cellulose, 2016, 23(3) : 1885-1897.

[15] ZAMANI F, AMANI-TEHRAN M, ZAMINY A, et al. Conductive 3D structure nanofibrous scaffolds for spinal cord regeneration[J]. Fibers \& Polymers, 2017, 18(10): 1874-1881.
作者简介: 杨昆, 女, 1993 年出生, 主要研究方向为复合材料 3D 打印、 微纳 3D 打印。

E-mail: 1419216282@qq.com

兰红波(通信作者), 男, 1970 年出生, 博士, 教授, 博士研究生导师。 主要研究方向为微纳尺度 $3 \mathrm{D}$ 打印、复合材料 $3 \mathrm{D}$ 打印、多材料 $3 \mathrm{D}$ 打印、 大面积纳米压印光刻、微纳制造等。

E-mail: hblan99@126.com 